\title{
Interstitial Hydrogen in Fe/V Superstructures: Lattice Site Location and Thermal Vibration
}

\author{
Kristina Komander®, Tuan Tran®, Jitendra Saha®, Marcos V. Moro®, Gunnar K. Pálsson®, \\ Max Wolff@, and Daniel Primetzhofer® \\ Department of Physics and Astronomy, Uppsala University, Box 516, SE-751 20 Uppsala, Sweden
}

(Received 11 June 2021; revised 13 August 2021; accepted 17 August 2021; published 24 September 2021)

\begin{abstract}
We report real space location of hydrogen in single crystalline Fe/V superstructures. Anisotropic strain is quantified versus hydrogen concentration by using the yield of backscattered primary $2 \mathrm{MeV}^{4} \mathrm{He}$ ions for incidence in different crystallographic directions. From a comparison of ion channeling in combination with ${ }^{1} \mathrm{H}\left({ }^{15} \mathrm{~N}, \alpha \gamma\right){ }^{12} \mathrm{C}$ nuclear reaction analysis and Monte Carlo simulations we show that hydrogen is located in octahedral $z$ sites and quantify its vibrational amplitude of $0.2 \AA$.
\end{abstract}

DOI: 10.1103/PhysRevLett.127.136102

Interstitial hydrogen in metals plays a crucial role in numerous fields such as hydrogen storage [1], hydrogen embrittlement [2], and high-temperature superconductivity [3]. Metal hydrides exhibit phase diagrams topologically analogous to fluids [4] while their thermodynamic properties are sensitive to effects of finite size and elastic strain due to the long-range of interactions between hydrogen atoms [5]. Requirements for storage applications are high hydrogen absorption, fast delivery at ambient temperatures, reversibility, and durability. Great benefit can be derived from nanostructures with improved reaction kinetics and reduced enthalpy of formation [6]. For example, octahedral palladium nanoparticles feature higher hydrogen loading compared to bulk or cubic nanoparticles [7]. Two-dimensional nanostructures like superlattices allow detailed exploration of the influence of finite size, interfaces, and strain on hydrogen absorption dynamics. By increasing the strain in niobium layers embedded in $\mathrm{Nb} / \mathrm{W}(110)$ superlattices hydrogen-hydrogen interactions can be tuned from being attractive to repulsive [8], similarly to changing elastic boundary conditions of vanadium from $\mathrm{Fe}$ to Mo $[9,10]$. In $\mathrm{Fe} / \mathrm{V}$ superlattices the critical temperature is observed to scale linearly with the inverse thickness of vanadium films [11]. A remarkable feature of vanadium hydride is the random distribution of hydrogen over 4T tetrahedral sites in the high temperature $\alpha$ phase or specific $\mathrm{O}$ octahedral sites in an ordered arrangement in the low temperature, high concentration $\beta$ phase [12]. Densityfunctional calculations imply the preference of site occupation is susceptible to biaxial compression [13]. However,

Published by the American Physical Society under the terms of the Creative Commons Attribution 4.0 International license. Further distribution of this work must maintain attribution to the author(s) and the published article's title, journal citation, and DOI. Funded by Bibsam. substantial evidence of the influence of nanostructuring on hydrogen site occupation is scarce.

As hydrogen is the most lightweight atomic element, both qualitative and quantitative experimental investigation of spatial distribution and occupancy at the atomic level encounter difficulties in ultrathin solid films and at interfaces [4]. Quantitative three-dimensional composition profiles of deuterium in needle-shaped samples can be reconstructed by atom probe tomography (APT) [14]. In reciprocal space, the interstitial position of deuterium in bulk samples can be refined from in situ neutron diffraction data [15] and distances between hydrogen atoms as well as vibrational modes can be accessed via inelastic scattering of neutrons [16]. Three-dimensional electron diffraction and a structure refinement algorithm can be used to locate hydrogen in bare surface nanocrystals [17]. Advances in most recent years allow imaging of the interstitial site with atomic-scale resolution by aberration-corrected scanning transmission electron microscopy (STEM) utilizing integrated differential phase contrast (iDPC) [18]. Nonetheless, metal hydride systems relevant for storage applications release hydrogen under the measurement conditions [19]. Nuclear reaction analysis (NRA) measures hydrogen at the surface and inside a film on absolute scales in real space [20]. Doppler spectroscopy reveals the zero-point vibrational motion of hydrogen bound to surfaces [21]. Ion channeling, within studies of transmission channeling through cooled, clean, freestanding, single crystalline foils [22] and nuclear reaction yields averaged over large bulk crystals $[23,24]$, has been utilized to detect the location of interstitial hydrogen and deuterium atoms. The effect is based on charged ion projectiles experiencing steering and blocking effects along major crystallographic axes and planes due to Coulomb repulsion [25].

In this Letter the interstitial site occupation and thermal vibration of hydrogen in strained $\mathrm{Fe} / \mathrm{V}$ superstructures are 
determined for the first time, by channeling ${ }^{15} \mathrm{~N}$ ions, and using the resonant ${ }^{1} \mathrm{H}\left({ }^{15} \mathrm{~N}, \alpha \gamma\right){ }^{12} \mathrm{C}$ nuclear reaction. This technique probes solely and quantitively the nanometerresolved depth distribution of hydrogen in a matrix. The anisotropic strain in vanadium under hydrogen loading is extracted from standard Rutherford backscattering spectrometry (RBS) in channeling geometry [26,27], while a computational approach uses ion tracing for numerical analysis.

Epitaxial single crystalline thin films grow on crystalline substrates, if the lattice mismatch between substrate and film is small. Strained V films are embedded in an Fe/V superlattice, clamped by epitaxial growth on single crystalline $\mathrm{MgO}(001)$ via dc magnetron sputtering [28]. Fully reversible exothermic absorption of hydrogen and concurrent volume expansion occur solely in the $\mathrm{V}$ layers since absorption is endothermic in Fe [29,30]. A bilayer has a nominal $\mathrm{Fe} / \mathrm{V}$-ratio of $2 / 14$ monolayers (ML) and is repeated 23 times. The sample is capped with $14 \mathrm{ML}$ of $\mathrm{V}$ and $7 \mathrm{~nm}$ of Pd to catalyze the dissociation of molecular hydrogen during hydrogen absorption [31] and to protect the sample from oxidation. Figure 1(a) shows a high-angle annular dark field STEM (HAADF-STEM) image along the [110] axis of the superlattice with discrete $\mathrm{Fe} / \mathrm{V}$ interfaces of negligible waviness. Atomic resolution reveals the excellent crystal quality in Fig. 1(b). To hinder potential hydrogen desorption during experiments, the sample is coated with $40 \mathrm{~nm}$ of $\mathrm{Al}_{2} \mathrm{O}_{3}$ deposited by reactive magnetron sputtering [32]. Hydrogenation is performed in a temperature-controlled high vacuum chamber. The sample is heated to $120^{\circ} \mathrm{C}$ and exposed to $100-500$ mbar of purified hydrogen. When equilibrium is reached the chamber is gradually evacuated while cooled to room temperature. After loading the absolute hydrogen concentration was determined by NRA measurements.

The RBS and NRA experiments were done at the $5 \mathrm{MV}$ 15 SDH-2 Tandem accelerator at Uppsala University, Sweden. Hydrogen is directly detected with resonant NRA using the nuclear reaction of ${ }^{15} \mathrm{~N}$ ions of $>6.385 \mathrm{MeV}$ in the laboratory frame resulting in characteristic $\gamma$ emission of $4.43 \mathrm{MeV}$. Fluences below $2 \mathrm{nA}$ prevent hydrogen leakage. Sequencing the ion energy up to 6.7 MeV allows hydrogen depth profiling because of the energy loss in the film. The resolution is $5-10 \mathrm{~nm}$ due to the width of the resonance of $1.8 \mathrm{keV}$, zero-point vibrational motion of the interstitial hydrogen atoms, energy distribution, and energy straggling of the ion projectiles [21,34]. The average concentration of hydrogen in vanadium is extracted from the depth profile, considering the iron content and normalization with respect to a H-implanted Si-standard, the material stopping power [35], and cumulated ion fluence.

The anisotropic strain in $\mathrm{Fe} / \mathrm{V}$ films is quantified by RBS-channeling experiments with primary $2 \mathrm{MeV}^{4} \mathrm{He}$ ions of $<10 \mathrm{nA}$. The ${ }^{4} \mathrm{He}$ ions backscattered after elastic

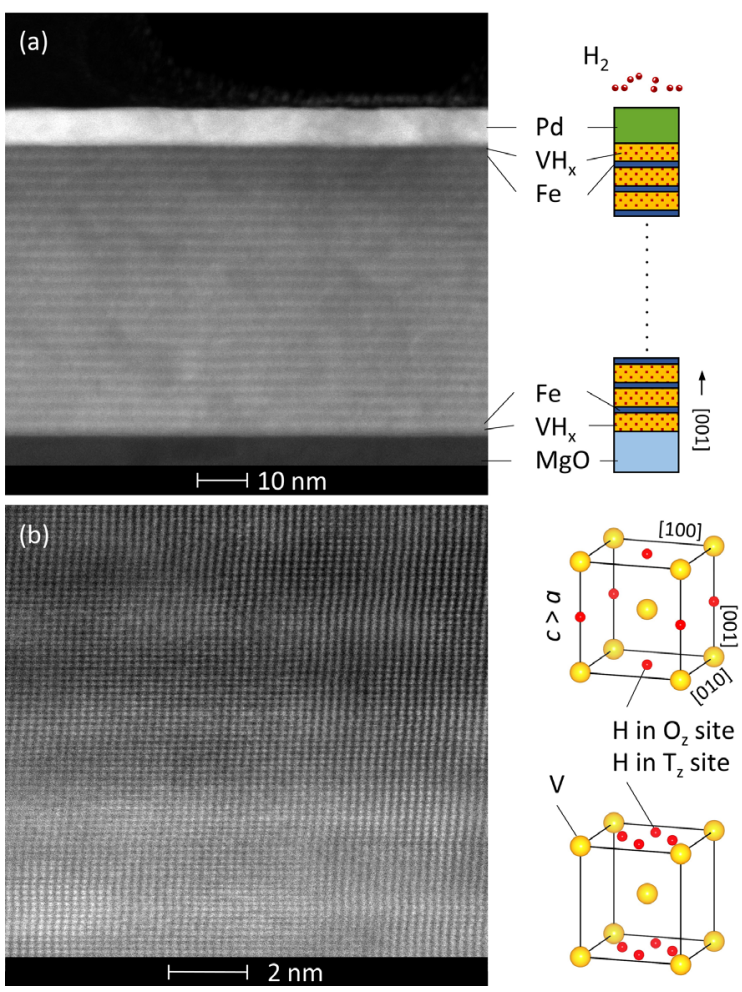

FIG. 1. (a) HAADF-STEM image showing the sample cross section of the superlattice along $<110>$, respectively, $<100>$ of $\mathrm{MgO}$, with $Z$-number contrast where lighter elements appear darker. The panel to the right sketches the schematic sample layout: $\mathrm{MgO} /[\mathrm{V} 14 \mathrm{ML} / \mathrm{Fe} 2 \mathrm{ML}] \times 23 / \mathrm{V} 14 \mathrm{ML} / \mathrm{Pd} 7 \mathrm{~nm}$. (b) Image of the single crystalline superlattice with atomic resolution. The right panel shows the tetragonal distorted bct vanadium unit cell with interstitial hydrogen occupying $\mathrm{T}_{z}$ or $\mathrm{O}_{z}$ sites [33].

collisions with constituent atoms were detected with a solid-state detector (SSD) at an angle of $\phi=170^{\circ}$ relative to the incident beam direction, as illustrated in Fig. 2(a). $\mathrm{Fe} / \mathrm{V}$ channeling maps are acquired by integrating the backscattered ion yield in the energy range from 1.2 to 1.6 $\mathrm{MeV}$ corresponding to collisions with $\mathrm{Fe} / \mathrm{V}$ atoms. The data are normalized to the primary ion fluence. The sample orientation (azimuth $\varphi$, tilt $\theta$ ) towards the incident beam is scanned to inclined crystal axes in the (010) plane, namely, [013], [012], [011], relative to the reference direction [001]. The angular shifts of the symmetry axes with respect to the bcc bulk structure $\Delta \varphi$ allow extraction of the ratio between out-of-plane and in-plane lattice parameter, $c / a$ [25]. Figure 2(a) presents an RBS-channeling pattern around the [012] channel of the hydrogenated superlattice. The position of the channeling dip as expected for a cubic structure is indicated by the dashed line. The measured dip is clearly shifted to lower angles, which relates to anisotropic lattice expansion mainly along the $z$ axis. The variation of self-trapping energy with elongation of $z$ axis implies that occupation is limited to $z$ sites $[13,36]$. In Fig. 2(b) the anisotropic lattice expansion was extracted, 


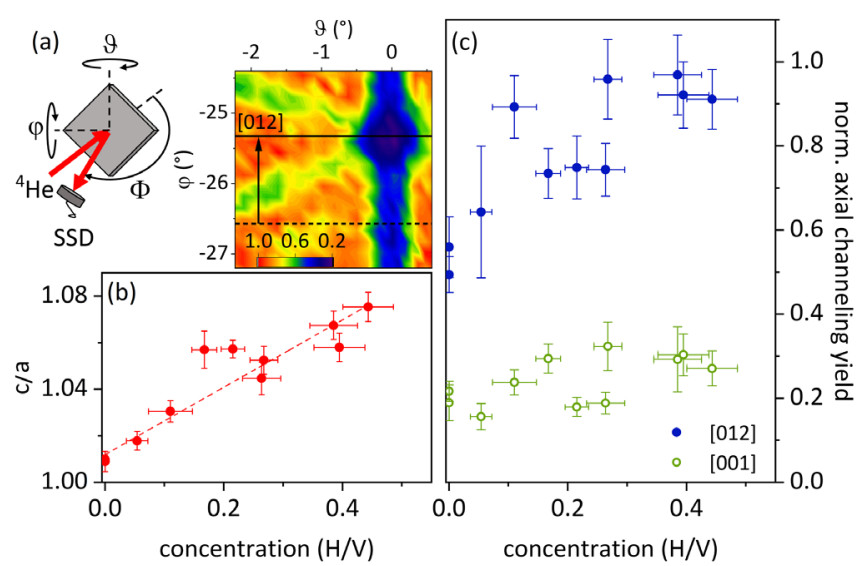

FIG. 2. (a) Schematic of the RBS geometry and angular map of primary $2 \mathrm{MeV}{ }^{4} \mathrm{He}$ ions backscattered from the hydrogenated $\mathrm{Fe} / \mathrm{V}$ superlattice around [012] orientation revealing the inclined [012] axis relative to bcc (dashed line). (b) Anisotropic expansion of the $\mathrm{Fe} / \mathrm{V}$ superlattice versus average hydrogen concentration. (c) Minimum axial channeling yield normalized by integrated Pd backscattering yield for different average hydrogen loads; (White filled circle), [001]; (Black filled circle), [012].

and the $c / a$ ratio is plotted versus the average hydrogen concentration. A linear expansion coefficient of $(0.14+/-0.01)$ can be fitted to the data. The $c / a$ ratio of the unhydrogenated superlattice is $(1.012+/-0.003)$ following registry with the $\mathrm{MgO}$ substrate and is consistent with literature data [28]. Figure 2(c) depicts the backscattering yield in the channel center versus hydrogen concentration. For the [012] orientation the yield increases demonstrating dechanneling with increasing hydrogen content. In contrast, for the [001] channel the channeling yield is independent of hydrogen concentration.

${ }^{4} \mathrm{He}$ ion trajectories through the $\mathrm{Fe} / \mathrm{V}$ superlattice are determined by binary collisions and the atomic-string approximation of the single crystal using the ZieglerBiersack-Littmark potential with Monte Carlo methodbased calculations by an updated version of the program FLUX7 [37]. Debye temperatures of $390 \mathrm{~K}$ and $373 \mathrm{~K}$ for $\mathrm{V}$ and Fe, respectively, are taken from Ref. [38]. Derived is the normalized average nuclear encounter probability (NEP) of ion projectiles to lattice atoms, proportional to the theoretical yield of nuclear interactions such as elastic backscattering (RBS) or nuclear reactions (NRA). Simulating the channeling of $2 \mathrm{MeV}{ }^{4} \mathrm{He}$ ions in [001] and [012] in an empty superlattice clamped in-plane to $2.98 \AA$ expanding uniaxially in vanadium from $c / a=1.01$ to 1.08 , reveals constant ${ }^{4} \mathrm{He}-\mathrm{NEP}$ with $\mathrm{Fe} / \mathrm{V}$ lattice atoms. Thus, expansion can be excluded as a cause for observed dechanneling. The supporting program YIMP7 determines the NEP with impurity sites in the crystal, such as vibrating interstitial hydrogen. Assuming a vibrational amplitude of $0.15 \AA$, the ${ }^{4} \mathrm{He}-\mathrm{NEP}$ to hydrogen exhibits differences for the possible interstitial locations. Occupation of $\mathrm{O}_{z}$ sites

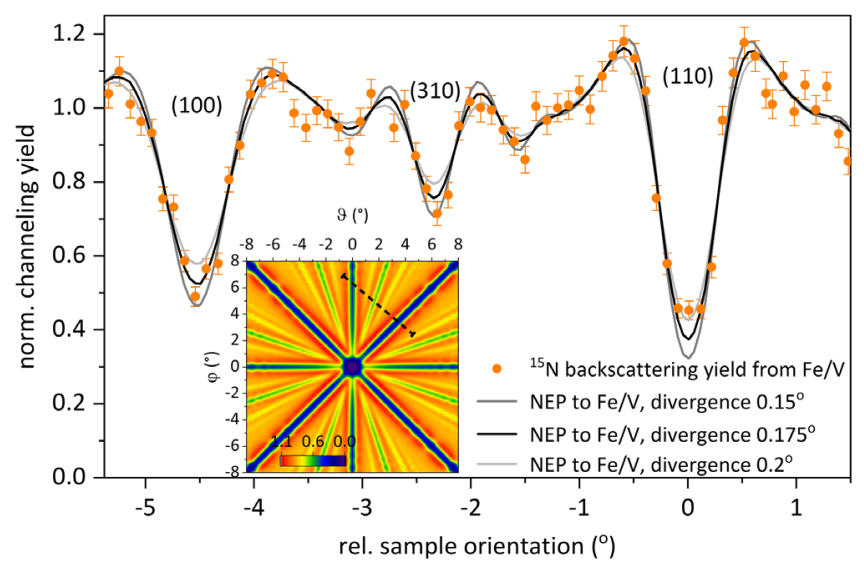

FIG. 3. Angular line scan of backscattering primary $6.55 \mathrm{MeV}$ ${ }^{15} \mathrm{~N}$ ions and comparison to simulation of average ${ }^{15} \mathrm{~N}-\mathrm{NEP}$ assuming different ion divergences in the lattice represented by the solid lines. (inset) Angular map of simulated average ${ }^{15} \mathrm{~N}-\mathrm{NEP}$ to $\mathrm{Fe} / \mathrm{V}$ atoms. The dotted line indicates the experimental angular line scan.

increases the interaction with steered ${ }^{4} \mathrm{He}$ projectiles in the [012] relative to the [001] channel from 0.13 to 0.53 , while interaction is highly probable in both directions with $\mathrm{T}_{z}$ site occupancy, 1.05 and 0.92 , respectively. As small-angle scattering from interstitials can result in the observed dechanneling effect, occupation of $\mathrm{O}_{z}$ sites explains decreased ion channeling along [012] with higher hydrogen load in Fig. 2(b).

Quantitative information about coordinates of interstitial hydrogen and its thermal vibrational amplitude is extracted by angular-resolved NRA measurements with ${ }^{15} \mathrm{~N}$ ions of $6.55 \mathrm{MeV}$. At this energy hydrogen is probed exclusively in the center of the $\mathrm{Fe} / \mathrm{V}$ superstructure. An SSD detects backscattered ${ }^{15} \mathrm{~N}$ ions at a scattering angle of $\phi=160^{\circ}$. Weakly bound hydrogen is expected to be sensitive to long ion exposure [34]. Therefore, alignment and measurement are performed simultaneously in a single linear scan by rotation of the sample around one axis, as marked by the dashed line in the inset of Fig. 3 and the top panels of Fig. 4. The sample orientation is derived from the distances between the planes without a need for biaxial alignment. This approach allows a probe of various projections of the impurities enabling unambiguous analysis of lattice site location and vibrational amplitude with low beam exposure.

The angular yield of ${ }^{15} \mathrm{~N}$ ions backscattered from $\mathrm{Fe} / \mathrm{V}$ during NRA and the comparison to the simulated ${ }^{15} \mathrm{~N}-\mathrm{NEP}$ is displayed in Fig. 3. The hydrogen content in the sample was $(0.44+/-0.03) \mathrm{H} / \mathrm{V}$. Channeling dips corresponding to (100), (310), and (111) planes are clearly visible. The minimum yield is primarily dependent on the initial beam divergence, and to a lesser degree straggling in the capping layers, crystal defects and phonon softening $[39,40]$, which are summarized in one single parameter in our study. Thus, 

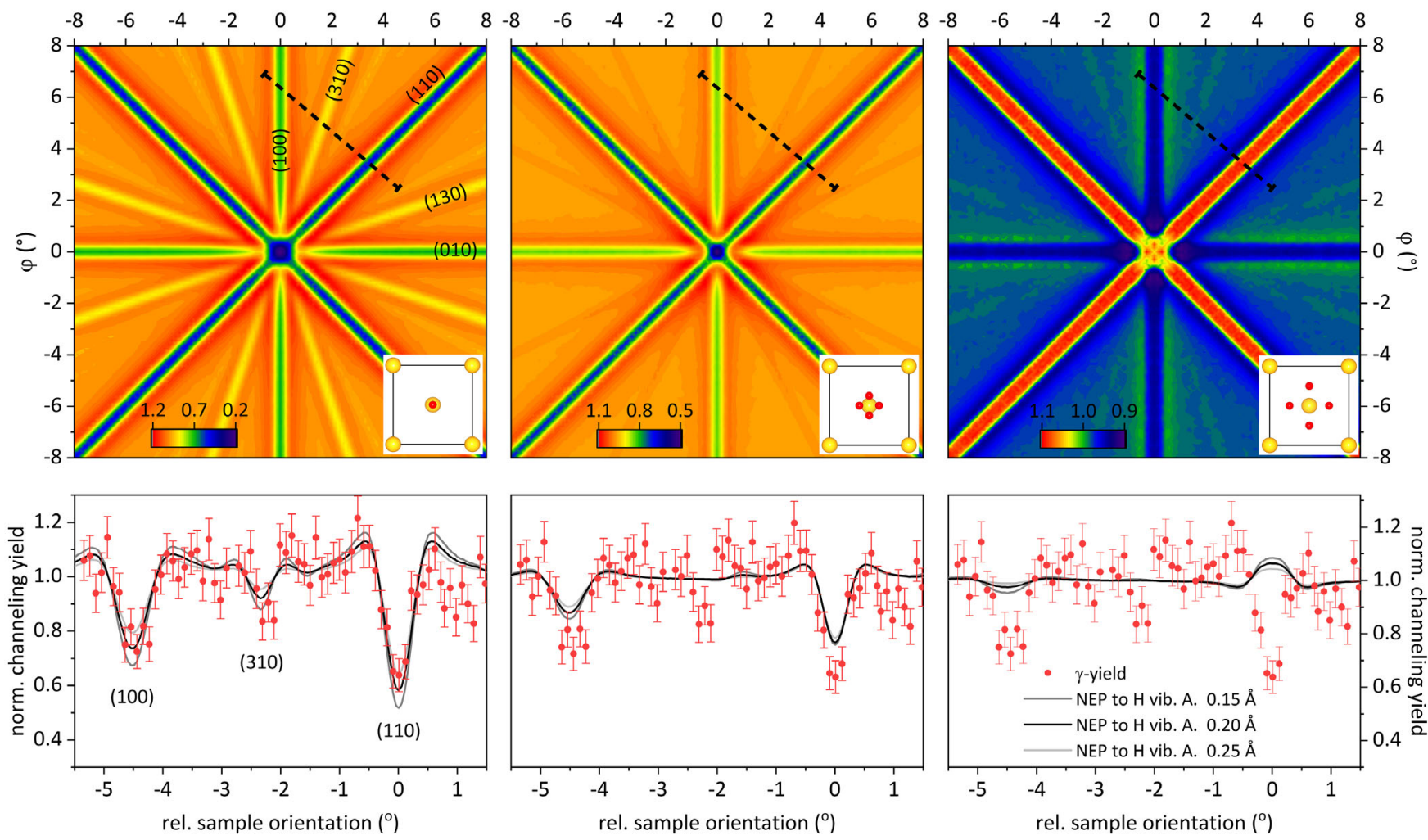

FIG. 4. (Upper panels) Simulated NEP for $6.55 \mathrm{MeV}$ primary ${ }^{15} \mathrm{~N}$ ions steered through the $\mathrm{Fe} / \mathrm{V}$ superlattice to hydrogen atoms in $\mathrm{O}_{z}$ (left), between $\mathrm{O}_{z}$ and $\mathrm{T}_{z}$ sites (middle), and $\mathrm{T}_{z}$ sites (right). Incident beam angles are varied around the [001] channel. (insets) The location of hydrogen atoms (red) in the V unit cell (yellow) for a projection along [001] [33]. (Lower panels) Normalized $\gamma$ yield (red) from the angular line scan (dashed black line in upper viewgraphs) is compared to the NEP for different thermal vibrational amplitudes of hydrogen (continuous lines).

using the derived effective ion divergence of $0.175^{\circ}$, the hydrogen site location and vibrational amplitude can be extracted.

The upper panels of Fig. 4 show simulated angular maps of ${ }^{15} \mathrm{~N}-\mathrm{NEP}$ with vibrating hydrogen atoms displaced stepwise from the $\mathrm{O}_{z}$ to the $4 \mathrm{~T}_{z}$ site (left to right). The position of the hydrogen atom is indicated schematically in the insets. The lower panels of Fig. 4 present the measured $\gamma$ yield from nuclear reactions with hydrogen. The dips are related to shadowing of hydrogen in atomic rows of the host lattice from steered primary ${ }^{15} \mathrm{~N}$ ions, as attenuation of $\gamma$ rays is very low. The occupation of $4 \mathrm{~T}_{z}$ is readily excluded upon comparison to the ${ }^{15} \mathrm{~N}-\mathrm{NEP}$-pattern for the angular line scan, indicated by solid lines. Considering the $\gamma$ pattern of (100) and (110) planes, $\mathrm{O}_{z}$ and displaced sites are possible but the observation of the (310) symmetry clearly confirms $\mathrm{O}_{z}$ location. Ab initio calculations [13] predicted energetically favored $\mathrm{O}_{z}$ sites for initial anisotropic strain of $c / a>1.043$ and above a critical concentration of $(0.28+/-0.07) \mathrm{H} / \mathrm{V}$ due to increased spacing between vanadium atoms close to interstitial hydrogen atoms. The minimum $\gamma$ yield decreases with smaller thermal vibrational amplitude as predicted by Lindhard's model [41]. The best overlap with the simulated ${ }^{15} \mathrm{~N}-\mathrm{NEP}$ is found for an amplitude of $0.2 \AA$.

We show that hydrogen is localized at $\mathrm{O}_{z}$ interstitial sites in uniaxially expanding vanadium hydride films of initial strain $c / a=(1.012+/-0.003)$ with a concentration of $(0.44+/-0.03) \mathrm{H} / \mathrm{V}$ at $295 \mathrm{~K}$. The result is extracted from ${ }^{15} \mathrm{~N}$ channeling in combination with simulations and axial dechanneling of steered ${ }^{4} \mathrm{He}$ ions along [012]. While quantitative analysis based on trajectory simulations in an empty lattice is straight forward for ${ }^{15} \mathrm{~N}$ ions, the analysis of experiments with light projectiles or recoils such as standard RBS or H-ERD will be affected by the neglection of interstitial hydrogen. Small-angle scattering from ordered interstitial impurities affects the light ion channeling yield, which is not considered in the calculations. Because of the high sensitivity, resolution, and the nondestructive, quantitative nature of the presented ${ }^{15} \mathrm{~N}$ method, it offers opportunities to investigate in situ the fundamental behavior of hydrogen absorption below surfaces on the atomic level within nanocrystals, thin films, multilayers, and clusters of metal-metal hydrides. 
The authors gratefully acknowledge access to the Tandem accelerator infrastructure by the Swedish Research Council VR-RFI (Contract No. 2017-00646_9) as well as the Swedish Foundation for Strategic Research (SSF) under Contract No. RIF14-0053.

*kristina.komander@physics.uu.se

[1] L. Schlapbach and A. Züttel, Nature (London) 414, 353 (2001).

[2] R. A. Oriani, Annu. Rev. Mater. Sci. 8, 327 (1978).

[3] M. Somayazulu, M. Ahart, A. K. Mishra, Z. M. Geballe, M. Baldini, Y. Meng, V. V. Struzhkin, and R. J. Hemley, Phys. Rev. Lett. 122, 027001 (2019).

[4] A. Pundt and R. Kirchheim, Annu. Rev. Mater. Res. 36, 555 (2006).

[5] A. Pundt, Adv. Eng. Mater. 6, 11 (2004).

[6] V. Bérubé, G. Radtke, M. Dresselhaus, and G. Chen, Int. J. Energy Res. 31, 637 (2007).

[7] A. Zalineeva, S. Baranton, C. Coutanceau, and G. Jerkiewicz, Sci. Adv. 3, e1600542 (2017).

[8] S. Olsson, B. Hjörvarsson, E. B. Svedberg, and K. Umezawa, Phys. Rev. B 66, 155433 (2002).

[9] G. Andersson, B. Hjörvarsson, and P. Isberg, Phys. Rev. B 55, 1774 (1997).

[10] G. Reynaldsson, S. Olafsson, and H. P. Gislason, J. Alloys Compd. 356-357, 545 (2003).

[11] X. Xin, G. K. Pálsson, M. Wolff, and B. Hjörvarsson, Phys. Rev. Lett. 113, 046103 (2014).

[12] G. Alefeld, Ber. Bunsenges. Phys. Chem. 76, 746 (1972).

[13] R. Johansson, R. Ahuja, O. Eriksson, B. Hjörvarsson, and R. H. Scheicher, Sci. Rep. 5, 10301 (2015).

[14] Y. S. Chen, H. Lu, J. Liang, A. Rosenthal, H. Liu, G. Sneddon, I. McCaroll, Z. Zhao, W. Li, A. Guo, and J. M. Cairney, Science 367, 171 (2020).

[15] A. Machida, H. Saitoh, H. Sugimoto, T. Hattori, A. Sano-Furukawa, N. Endo, Y. Katayama, R. Iizuka, T. Sato, M. Matsuo, S. I. Orimo, and K. Aoki, Nat. Commun. 5, 5063 (2014)

[16] A. Borgschulte, J. Terrenia, E. Billetera, L. Daemenc, Y. Cheng, A. Pandey, Z. Łodzianad, R. J. Hemleye, and A. J. Ramirez, Proc. Natl. Acad. Sci. U.S.A. 117, 4021 (2020).

[17] L. Palatinus, P. Brázda, P. Boullay, O. Perez, M. Klementová, S. Petit, V. Eigner, M. Zaarour, and S. Mintova, Science 355, 166 (2017).

[18] S. de Graaf, J. Momand, C. Mitterbauer, S. Lazar, and B. J. Kooi, Sci. Adv. 6, eaay4312 (2020).

[19] S. V. Alapati, J. K. Johnson, and D. S. Sholl, J. Phys. Chem. B 110, 8769 (2006)
[20] J. F. Ziegler, C. P. Wu, P. Williams, C. W. White, B. Terreault, B. M. U. Scherzer, R. L. Schulte, E. J. Schneid, C. W. Magee, E. Ligeon et al., Nucl. Instrum. Methods 149, 19 (1978).

[21] K. Fukutani, A. Itoh, M. Wilde, and M. Matsumoto, Phys. Rev. Lett. 88, 116101 (2002).

[22] I. Stensgaard and F. Jakobsen, Phys. Rev. Lett. 54, 711 (1985).

[23] H. D. Carstanjen, Phys. Status Solidi A 59, 11 (1980).

[24] E. Yagi, T. Hayashi, T. Yoshida, S. Koike, N. Higami, K. Hirabayashi, A. Takebayashi, H. Muba, and K. Ogiwara, J. Phys. Soc. Jpn. 81, 044602 (2012).

[25] L. C. Feldman, J. W. Mayer, and S. T. Picraux, Materials Analysis by Ion Channeling-Submicron Crystallography (Academic Press, New York 1982).

[26] K. Komander, M. Moro, J. Saha, M. Wolff, and D. Primetzhofer, Nucl. Instrum. Methods Phys. Res., Sect. B 486, 63 (2021).

[27] S. T. Picraux, W. K. Chu, W. R. Allen, and J. A. Ellison, Nucl. Instrum. Methods Phys. Res., Sect. B 15, 306 (1986).

[28] S. A. Droulias, G. K. Pálsson, H. Palonen, A. Hasan, K. Leifer, V. Kapaklis, B. Hjörvarsson, and M. Wolff, Thin Solid Films 636, 608 (2017).

[29] R. Griessen and T. Riesterer, Top. Appl. Phys. 63, 219 (1988).

[30] J. Bloch, B. Hjörvarsson, S. Olsson, and R. Brukas, Phys. Rev. B 75, 165418 (2007).

[31] F. Wang, R. Li, C. Ding, W. Tang, Y. Wang, S. Xu, R. Yu, and Z. Wang, Int. J. Hydrogen Energy 42, 11510 (2017).

[32] Y. Wang, G. K. Pálsson, H. Raanaei, and B. Hjörvarsson, J. Alloys Compd. 464, L13 (2008).

[33] K. Momma and F. Izumi, J. Appl. Crystallogr. 44, 1272 (2011).

[34] M. Wilde and K. Fukutani, Surf. Sci. Rep. 69, 196 (2014).

[35] J. F. Ziegler, SRIM 2008 (available from http://www.srim .org).

[36] H. Sugimoto, J. Phys. Soc. Jpn. 53, 2592 (1984).

[37] P. J. M. Smulders and D. O. Boerma, Nucl. Instrum. Methods Phys. Res., Sect. B 29, 471 (1987).

[38] C. Y. Ho, R. W. Powell, and P. E. Liley, J. Phys. Chem. Data 3, Suppl. 1 (1974).

[39] S. Kim, H. Suhl, and I. K. Schuller, Phys. Rev. Lett. 78, 322 (1997).

[40] M. Zimmermann, G. Wolf, and K. Bohmhammel, Phys. Status Solidi A 31, 511 (1975).

[41] J. Lindhard, Mat. Fys. Medd. K. Dan. Vidensk. Selsk 34, 14 (1965). 